\title{
What is the role of adipokines in myasthenia gravis?
}

This article was published in the following Dove Press journal:

Therapeutics and Clinical Risk Management

\author{
Izabela Rozmilowska' \\ Damian Czyzewski \\ Bogdan Mazur ${ }^{3}$ \\ Monika Adamczyk-Sowa' \\ 'Department of Neurology, Medical \\ University of Silesia, Zabrze, Poland; \\ ${ }^{2}$ Department of Thoracic Surgery, \\ Medical University of Silesia, Zabrze, \\ Poland; ${ }^{3}$ Department of Microbiology, \\ Medical University of Silesia, Zabrze, \\ Poland
}

Objective: Myasthenia gravis (MG) is a disease with autoimmune etiology. The main pathomechanism is related to the production of antibodies against nicotinic acetylcholine receptor. The present study is aimed to compare the serum level of adipokines in patients with MG with that in controls, as well as to study the relation of these levels with disease severity. Patients and methods: Fifty patients with MG and 30 healthy individuals were enrolled in our study. Serum concentrations of select adipokines, namely adiponectin, leptin, omentin, visfatin, and resistin were measured.

Results: The results showed a significant increase in serum concentrations of adiponectin and resistin in the patients with MG compared with the controls.

Conclusion: Further studies are warranted to assess changes in adipokine concentration levels in patients with MG.

Keywords: adiponectin, leptin, resistin, visfatin, omentin

\section{Introduction}

Myasthenia gravis (MG) is an acquired, chronic autoimmune disease characterized by a rapid increase in skeletal muscle weakness. It has a worldwide prevalence of 40-180 per 1,000,000 individuals, with an annual incidence rate of 4-12 per 1,000,000 individuals. ${ }^{1}$

The disease may occur at any age with two peaks: the first at $\sim 30$ years of age with female prevalence and the second at $\sim 60-70$ years of age with male prevalence. On the basis of the symptoms, MG is categorized into two main types: ocular MG (OMG) and generalized MG (GMG). The coexistence of other autoimmune diseases is observed in $30 \%$ of patients. ${ }^{2}$ Viral infections, stress, pregnancy, and puerperal period are some of the well-known factors that may predispose one to $\mathrm{MG}^{3}$

Approximately $80 \%-85 \%$ of patients have detectable antibodies against the nicotinic acetylcholine receptor (AChR), which leads to a reduction in the number of AChR molecules in the postsynaptic membrane of the neuromuscular junction. ${ }^{4}$ In some patients, the presence of muscle-specific kinase antibodies and antibodies against low-density lipoprotein receptor-related protein 4 is also observed. ${ }^{5,6}$

The management of MG is based on improving the efficiency of the neuromuscular junction by using cholinergic drugs. In case of ineffective treatment, the second-line therapy usually involves the additional use of corticosteroids, immunosuppressants, and in some patients, monoclonal antibodies (eg, rituximab). In cases of myasthenic crisis, rapid improvement can be achieved by using intravenous infusions of immunoglobulins or plasmapheresis. In addition to the pharmacological treatment, thymectomy is considered a proven and effective method of long-term treatment, which is supported by the key role of the thymic tissue in the pathogenesis of MG. Immunoregulatory defects in patients with MG are observed in both regulatory and effector $\mathrm{T}$ cells.
Correspondence: Izabela Rozmilowska Department of Neurology, Medical University of Silesia, ul. 3-go Maja I3-15, 4I-800 Zabrze, Poland

Tel +4832792049005

Fax +48 323704597

Email irozmilowska@gmail.com (c) 1 (1) (2) 2018 Rozmilowska et al. This work is published and licensed by Dove Medical Press Limited. The full terms of this license are available at https://www.dovepress.com/terms.php hereby accept the Terms. Non-commercial uses of the work are permitted without any further permission from Dove Medical Press Limited, provided the work is properly attributed. For permission for commercial use of this work, please see paragraphs 4.2 and 5 of our Terms (https://www.dovepress.com/terms.php). 
The natural regulatory $\mathrm{T}$ cells that differentiate in the thymus are clearly less functional in the thymus of patients with MG. This phenomenon is also observed in the peripheral parts of this organ. However, effector T cells in the thymus are resistant to suppression by regulatory T cells. ${ }^{7,8}$ This phenomenon is associated with changes in the expression of proinflammatory cytokines produced by $\mathrm{T}$ cells in MG patients, for example, IL-17, interferon-gamma, and IL-21. These data suggest that the inflammatory environment in the MG thymus changes the function and plasticity of $\mathrm{CD}^{+} \mathrm{T}$ lymphocytes, which leads to impaired functions of regulatory $\mathrm{T}$ lymphocytes and effector T-cell resistance to suppression. ${ }^{9-11}$

The multidirectional action of adipokines (ie, protective in metabolic diseases and proinflammatory in autoimmune diseases) suggests that changes in the concentrations of adipokines may also be related to the pathophysiology of MG. ${ }^{12}$ The relationship between the production and effect of adipokines in MG has not been extensively investigated. So far, only a single study by Zhang et al has been published. They concluded that increased resistin concentration in serum correlated with the severity of symptoms in patients with GMG and the presence of thymoma. It is believed that the proinflammatory and anti-inflammatory effects of individual adipokines may influence the course of $\mathrm{MG}$, both indirectly and directly, affecting the functioning of the neuromuscular junction and the production of specific antibodies. $^{13}$

Adipose tissue was previously perceived merely as a reservoir of fat. Studies conducted at a molecular level, mainly in patients with metabolic syndrome, have changed this perception. The adipose tissue is now considered as a very active endocrine organ that produces inflammatory cytokines, enzymes, and nitric oxide. ${ }^{14}$ More importantly, it is also a source of peptide hormones (adipokines) such as adiponectin, leptin, visfatin, omentin, and resistin. Adipokines are biologically active agents, and perform immune and regulatory functions in the process of energy conversion in fat cells of mammals. Together with other substances produced by adipocytes, such as proinflammatory cytokine IL-6, tumor necrosis factor alpha (TNF- $\alpha$ ), and chemokines, adipokines affect the functions of macrophages and monocytes. Adipocytes also synthesize molecules such as Clq/TNF-related proteins, thus affecting innate immunity. ${ }^{15}$

It has been demonstrated that adipokines may have a protective effect in metabolic diseases such as atherosclerosis or diabetes, whereas they may exert a proinflammatory effect in autoimmune diseases associated with chronic inflammation. ${ }^{14,15}$ It has also been proven that a higher number of fat cells increases the production of proinflammatory cytokines and the level of C-reactive protein (CRP). ${ }^{12}$

Recent studies have shown that adipokines play a significant role in autoimmune diseases such as systemic lupus erythematosus (SLE) and rheumatoid arthritis (RA). Patients with SLE often demonstrate metabolic changes that may be due to the disease, genetic background, or treatment. De Sanctis et al observed that the concentration of leptin was lower in the group of patients with SLE than in the control group, but the concentrations of adiponectin, ghrelin, and visfatin were higher in patients with SLE. Adipokine levels were independent of treatment. ${ }^{16}$ Similar results were obtained in a meta-analysis by Zhang et al, which showed a higher plasma level of adiponectin in patients with RA and SLE compared with healthy individuals. Because adiponectin is known to have potent anti-inflammatory properties, high adiponectin levels in patients with systemic autoimmune disease may alleviate the inflammatory response. However, it is necessary to determine the exact consequences of increased serum adiponectin concentration and its influence on the development of the metabolic syndrome and the risk of atherosclerotic cardiovascular diseases. ${ }^{17}$

Observations on leptin are ambiguous. Li et al did not show a significant difference in serum leptin levels between the group of SLE patients and the control group. However, it was noted that serum leptin levels were significantly higher in the Asian population subgroup of patients with SLE $($ aged $>40$ years and body mass index $[\mathrm{BMI}]<25){ }^{18}$ Additionally, Wislowska et al did not report differences between SLE patients and healthy subjects. However, a correlation was found between BMI and serum leptin levels in both the groups. Serum leptin concentrations in patients with SLE with arthritis and central nervous system involvement were significantly lower compared with patients with SLE but without arthritis and central nervous system involvement. Perhaps, this suggests that active chronic inflammation may decrease leptin plasma concentration levels. ${ }^{19}$

The studies on the involvement of resistin in SLE and RA seem to indicate its proinflammatory properties. The levels of resistin concentration measured by Almehed et al were not different between patients with SLE and the control group. However, it was shown that resistance to treatment was due to general inflammation, kidney disease, glucocorticoid treatment, and bone mass loss. ${ }^{20}$ Slightly different results were observed in patients with RA. Huang et al conducted a metaanalysis of available studies, which confirmed that serum resistin levels were significantly elevated in patients with RA. ${ }^{21}$

In addition to adipocytes, adiponectin is also produced in skeletal muscles and epithelial cells. High molecular weight 
adiponectin has been shown to induce IL-6 secretion, while the low molecular weight form results in a decreased production of IL- 6 and inhibition of IL-10 synthesis, thus increasing the anti-inflammatory effect. ${ }^{22}$

Leptin is a cytokine presenting proinflammatory properties. Its main activity is related to appetite control, modulation of the immune response and inflammation, functioning of the neuroendocrine system, energy homeostasis, hematopoiesis, and angiogenesis. Leptin results in an increased production of proinflammatory cytokines such as TNF- $\alpha$, IL-6, and epithelial cell growth factor. It also stimulates chemotaxis and synthesis of reactive oxygen molecules. Furthermore, it affects the proliferation of natural killer cells and T cells. ${ }^{23}$

Resistin is produced in the pancreas, adipocytes, muscles, and macrophages. Stimulation of resistin gene expression is mediated by proinflammatory cytokines such as TNF- $\alpha$, IL-6, and lipopolysaccharides. Its main action is to increase insulin resistance, and hyperglycemic and proinflammatory effect. It also inhibits adipocyte differentiation and maturation. ${ }^{24}$

Visfatin, known as pre-B-cell colony-enhancing factor 1 , is an enzyme of the nicotinamide adenine dinucleotidepositive reserve biosynthetic pathway. Studies confirmed that visfatin exerts a multidirectional effect; that is, it stimulates the production of proinflammatory cytokines (IL-1, IL-6, TNF- $\alpha$ ), causes a decrease in the number of apoptotic cells, and influences the process of proliferation, angiogenesis, and invasion of tumor cells. Additionally, it intensifies oxidative stress, stimulates differentiation of pre-B lymphocytes, and intensifies leukocyte transmigration. ${ }^{25}$

Omentin is mainly produced in adipocytes. In smaller amounts, it is also produced in the small intestine, lungs, and heart. Its biological functions are largely unknown. Recent reports have indicated that it may have a protective role in innate mechanisms of the immune response. It probably participates in the defense processes against bacterial intestinal infections. ${ }^{26}$

The aim of this study was to assess the role of select adipokines in the pathophysiology of $\mathrm{MG}$, which has not been investigated yet. The identification of the causative agent or a factor affecting the course of the disease and the effectiveness of treatment would be crucial elements in improving the diagnosis and therapy of patients with $\mathrm{MG}$.

\section{Patients and methods Ethical permission}

Fifty patients diagnosed with MG (age $60.7 \pm 12.4$ years) were enrolled in the study. The study participants were recruited from the Professor Stanisław Szyszko Independent Public Clinical Hospital No 1 in Zabrze, Poland. The control group consisted of 30 age- and gender-matched healthy volunteers (age $53.8 \pm 11.0$ years). All participants were informed about the study design, and written informed consent was obtained from all participants. The project was accepted by the Bioethics Committee of the Medical University of Silesia, Katowice, Poland (no KNW/0022/ KB1/68/15 of 7 July 2015).

\section{Patients}

History was obtained from all patients and healthy individuals participating in the study. Physical examination was performed by a neurologist. Additional electrodiagnostic evaluation included electromyography. The following anthropometric features were measured and estimated: height, weight, and BMI. History of thymoma, atrophic thymus, and thymectomy was considered. Furthermore, the patients were assessed using three selected scales used in the treatment of patients with MG: Myasthenia Gravis Composite (MG Composite), Myasthenia Gravis-Specific Activities of Daily Living, and Myasthenia Gravis Quality of Life 15 (MG-QOL-15). ${ }^{27-29}$ Table 1 presents more information on the clinical characteristics of the patients.

\section{Inclusion and exclusion criteria}

The study participants were selected according to specific criteria. The inclusion criteria were as follows: diagnosis

Table I Clinical characteristics of patients with MG

\begin{tabular}{ll}
\hline Patients with MG (n) & $\mathbf{5 0}$ \\
Mean duration of disease (years) & 9.48 \\
Average of disease (years) & $0.5-34.0$ \\
Early disease onset ( $\mathrm{n})$ & 21 \\
Late disease onset ( $\mathrm{n})$ & 29 \\
Gender & \\
Female (\%) & 56 \\
Male (\%) & 44 \\
Patients seropositive for AChR (\%) & 82 \\
Mean antibodies against AChR (nmol/L) & 8.79 \\
Average antibodies against AChR (nmol/L) & $0.1-85.0$ \\
Electrical activity in repetitive nerve stimulation (\%) & 86 \\
Mean BMI & 24.96 \\
Average BMI & $21.15-32.75$ \\
Main neurological symptoms & \\
Diplopy (\%) & 44 \\
Eyelids drooping (\%) & 72 \\
Difficulty chewing (\%) & 10 \\
Dysphagia (\%) & 16 \\
Dysarthria (\%) & 20 \\
Dyspnea (\%) & 4 \\
Apokamnosis symptoms of limbs and mimetics & 40 \\
muscles (\%) &
\end{tabular}

Abbreviations: MG, myasthenia gravis; AChR, acetylcholine receptor; BMI, body mass index. 
of $\mathrm{MG}$, disease duration of at least 6 months, and age 18-85 years. The patients were also randomized according to the type of MG (ocular or generalized form), level of symptom intensity, level of antibodies against nicotinic $\mathrm{AChR}$ and the regimen of previous treatment, and the absence of concomitant autoimmune diseases (eg, psoriasis, Crohn's disease, colitis ulcerosa, connective tissue disease, or Hashimoto's thyroiditis).

The exclusion criteria were as follows: age $<18$ years, suspected or diagnosed Lambert-Eaton myasthenic syndrome, diabetes mellitus, neoplastic disease (active or present within the last 5 years), obesity (BMI > 30), dementia, mental disorders, active infection, history of cerebrovascular insult, and administration of antioxidants or vitamins during the last 3 months.

In addition, patients were divided into groups based on the following: the level of AChR antibodies, disease duration, mediastinal neuroimaging assessment, time after thymectomy, duration of treatment with acetylcholinesterase inhibitors, and the clinical condition according to the Myasthenia Gravis Foundation of America (MGFA) clinical classification. $^{30}$

\section{Data collection}

Blood samples were collected from all study participants. A total of $15 \mathrm{~mL}$ of venous blood was collected from each patient $(5 \mathrm{~mL}$ was used for immediate basic biochemical and blood count analyses, while the remaining $10 \mathrm{~mL}$ was centrifuged; the serum samples were frozen at $-80^{\circ} \mathrm{C}$ until further use). Serum concentrations of select adipokines, namely adiponectin, leptin, omentin, visfatin, and resistin, were determined using the ELISA kit in compliance with the manufacturer's instructions (BioVendor). The measurements were repeated three times in order to confirm the obtained results. In the Adiponectin ELISA, standards, quality controls, and samples were incubated in microplate wells precoated with recombinant human adiponectin together with polyclonal antihuman adiponectin antibody conjugated to horseradish peroxidase (HRP). After the washing step, the HRP conjugate bound to the adiponectin immobilized on the wells was allowed to react with the substrate solution $\left(3,3^{\prime}, 5,5^{\prime}\right.$ tetramethylbenzidine). The reaction was stopped by adding acidic solution, and absorbance of the resulting yellow product was measured. The absorbance was found to be inversely proportional to the adiponectin concentration $(450 \mathrm{~nm})$. A standard curve was constructed by plotting absorbance values against adiponectin standards, and concentrations of unknown samples were determined using this curve.
Thawed samples were mixed thoroughly immediately before the assay. Samples were diluted $30 \times$ with dilution buffer prior to the assay, for example, $10 \mu \mathrm{L}$ of sample + $290 \mu \mathrm{L}$ of dilution buffer for duplicates.

The measured concentration of samples and quality controls calculated from the standard curve had to be multiplied by a dilution factor of 10 because standards were diluted $3 \times$ and samples and quality controls were diluted $30 \times$, for example, $1.05 \mu \mathrm{g} / \mathrm{mL}$ (from standard curve) $\times 10$ (dilution factor $)=10.5 \mu \mathrm{g} / \mathrm{mL}$.

\section{Statistical analysis}

Statistical analysis was performed using Excel and Statistica 12 PL by StatSoft. Data are presented as mean \pm SD or median and interquartile range. The Shapiro-Wilk test was used to evaluate the normality of the distribution. Data were analyzed using the Mann-Whitney $U$-test, Kruskal-Wallis test, and post hoc analysis. Pearson correlation analysis was performed by calculating the Pearson correlation coefficient. Statistical hypotheses were verified based on the significance level $p<0.05$.

\section{Results}

Fifty patients with MG aged $60.7 \pm 12.4$ years and 30 healthy individuals aged $53.8 \pm 11.0$ years were recruited from the Outpatient Neurology Clinic. No statistically significant differences were found in gender or age between patients with MG and controls ( $p=0.724$ and 0.219$)$.

The mean duration of the disease was $9.48 \pm 6.59$ years, and the mean level of AChR antibodies was $8.79 \pm 8.40 \mathrm{nmol} / \mathrm{L}$. In total, $82 \%$ of patients were AChR antibody positive and $18 \%$ were AChR antibody negative.

Ninety-eight percent of patients were treated with pyridostigmine bromide. The usual adult dose was 0.5-2 tablets (30-120 mg) taken three to six times daily. A total of $16 \%$ of patients were administered steroids, and $6 \%$ were given azathioprine.

The average BMI was 24.96 (range 21.15-32.75) in the patients with $\mathrm{MG}$, while it was 22.85 in the healthy individuals - the differences did not show statistical significance. Tables 2 and 3 present the detailed characteristics of the patients with MG. The concentrations of the analyzed adipokines in patients with early-onset diagnosis $(n=21)$ and late-onset diagnosis of MG $(n=29)$ were compared. No statistically significant differences were observed between concentrations of the following: adiponectin $(p=0.347)$, omentin ( $p=0.912)$, visfatin $(p=0.058)$, lectin $(p=0.255)$, and resistin $(p=0.152)$. Table 4 shows the data on serum 
Table 2 Demographic characteristics of patients qualified for the survey

\begin{tabular}{ll}
\hline Patients with myasthenia gravis (n) & $\mathbf{5 0}$ \\
Gender & \\
Female (\%) & 56 \\
Male (\%) & 44 \\
Mean age (years) & $60.66 \pm 12.41$ \\
Age range (years) & $30-8 \mathrm{I}$ \\
Healthy control group (n) & 30 \\
Mean age (years) & $53.8 \pm 11 . \mathrm{I}$ \\
Age range (years) & $32-76$ \\
Gender & \\
Female (\%) & 57 \\
Male (\%) & 43 \\
\hline
\end{tabular}

Abbreviation: $\mathrm{n}$, number of patients.

concentrations of adipokines in different sample subgroups and the correlation data.

In the performed analysis, the obtained median levels of adiponectin were higher in the patients with MG compared with the healthy control group, and this difference showed statistical significance at $p<0.001$. On average, the adiponectin concentration was 2.5 times higher. Detailed information is presented in Table 5 and Figure 1.

Numerous relationships were found between the concentrations of adipokines in the serum of patients with MG and the selected clinical factors. A positive correlation was observed between the concentration of adiponectin and the form of MG, the intensity of the symptoms of apokamnosis, and the concentration of IL-33. Patients with GMG had statistically significantly higher adiponectin levels compared to patients with OMG ( $p<0.001, r=0.5983)$. It was shown that the intensity of limb muscle fatigue noted during neurological examination correlated positively with

Table 3 Detailed information on patients with MG

\begin{tabular}{ll}
\hline Type of MG & \\
Ocular (\%) & 48 \\
Generalized (\%) & 52 \\
Thymoma (\%) & 10 \\
Hyperplastic thymus (\%) & 56 \\
Thymectomy (\%) & 58 \\
Mean doses of anticholinesterase drugs (mg/day) & 197.8 \\
MGFA classification (\%) & \\
Remission & 22 \\
Class I (ocular) & 38 \\
Class II (mild, general) & 20 \\
Class III (moderate, general) & 14 \\
Class IV (severe, general) & 6 \\
Class V (very severe) & 0 \\
\hline
\end{tabular}

Abbreviations: MG, myasthenia gravis; MGFA, the Myasthenia Gravis Foundation of America.

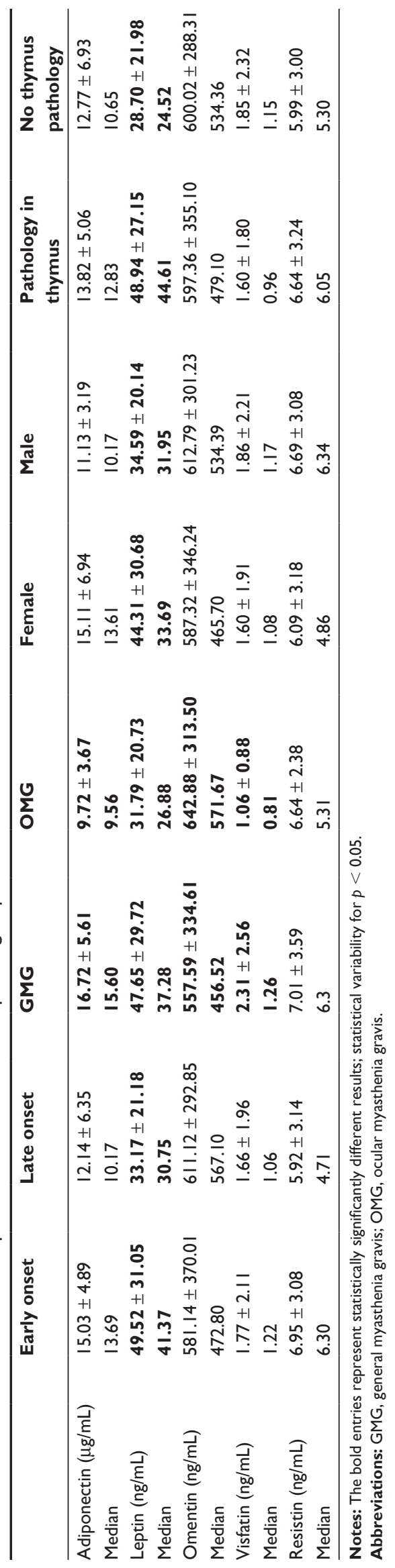


Table 5 Concentrations of select adipokines, namely adiponectin, leptin, omentin, visfatin, and resistin, in patients with MG and in healthy persons

\begin{tabular}{|c|c|c|c|}
\hline & $\begin{array}{l}\text { Patients with } \\
\text { MG }\end{array}$ & $\begin{array}{l}\text { Healthy control } \\
\text { group }\end{array}$ & $p$-value \\
\hline Adiponectin $(\mu \mathrm{g} / \mathrm{mL})$ & $13.36 \pm 5.90$ & $5.38 \pm 2.79$ & $<0.001$ \\
\hline Median (IQR) & II.87 (6.78) & $4.48(3.26)$ & $<0.00 \mathrm{I}$ \\
\hline Leptin (ng/mL) & $40.03 \pm 26.76$ & $39.50 \pm 26.36$ & ns \\
\hline Median (IQR) & 31.98 (37.72) & $28.67(16.19)$ & ns \\
\hline Omentin $(\mathrm{ng} / \mathrm{mL})$ & $598.53 \pm 324.21$ & $525.87 \pm 210.20$ & ns \\
\hline Median (IQR) & $489.58(421.08)$ & $505.00(275.92)$ & ns \\
\hline Visfatin (ng/mL) & $1.71 \pm 2.03$ & $1.20 \pm 1.22$ & ns \\
\hline Median (IQR) & I.II (I.I4) & $0.88(\mathrm{I} .1 \mathrm{I})$ & ns \\
\hline Resistin (ng/mL) & $6.35 \pm 3.12$ & $4.39 \pm 1.14$ & $<0.001$ \\
\hline Median (IQR) & $6.00(3.88)$ & $4.20(1.80)$ & $<0.001$ \\
\hline
\end{tabular}

Notes: The results are expressed as arithmetic mean \pm SD; statistical variability for $p<0.05$.

Abbreviations: MG, myasthenia gravis; IQR, interquartile range; ns, nonsignificant.

the level of adiponectin $(p<0.001 ; r=0.5329)$. A positive correlation was also found between adiponectin concentration and female gender, a higher dose of acetylcholinesterase inhibitors, MG-QOL-15 score, MG Composite score, and patients with MGFA class IIIb, according to the severity of the disease. A negative correlation was observed between BMI level and adiponectin concentration in the serum of patients with MG ( $p=0.004 ; r=-0.3961)$. Figures $2-6$ report serum concentration values of adiponectin in patients with MG classified on the basis of clinical features.

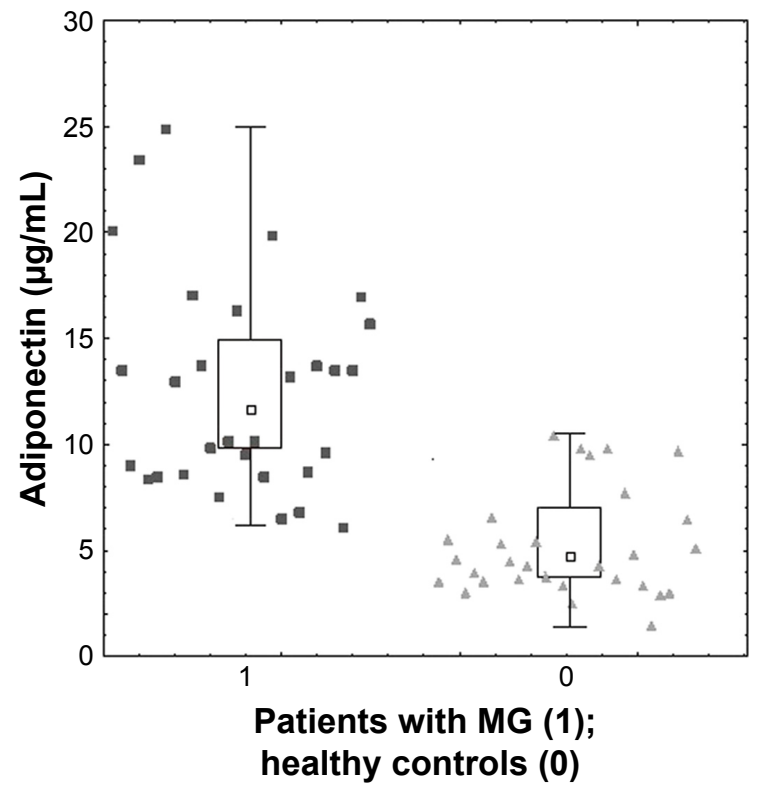

Post hoc analysis of adiponectin concentration changes in the serum of patients with MG in relation to the clinical condition of patients according to the MGFA classification showed a statistically significantly higher adiponectin concentration in patients with moderate clinical symptoms (MGFA class IIIb) compared with patients with mild clinical symptoms (MGFA classes I and IIa; Table 6).

Statistical analysis showed a statistically significantly positive relationship between visfatin concentration and CRP level $(p<0.001 ; r=4.966)$. The only statistically significant relationship was observed between AChR antibody concentration and visfatin concentration. However, the correlation was low $(p=0.015 ; r=0.3429)$.

In the performed analysis, the obtained median levels of resistin were higher in the patients with MG compared with the healthy control group, and this difference showed statistical significance at $p<0.001$ (Figure 1). The relationships were found between the concentrations of resistins in the serum of patients with MG and the selected clinical factors. A positive correlation was obtained between resistin concentration and the dose of acetylcholinesterase inhibitors $(p=0.003 ; r=0.4102)$.

In the case of leptin, a strong positive correlation was observed between leptin concentration and the duration of disease $(p<0.001 ; r=6.313)$, the duration of treatment with acetylcholinesterase inhibitors $(p<0.001 ; r=0.8062)$,

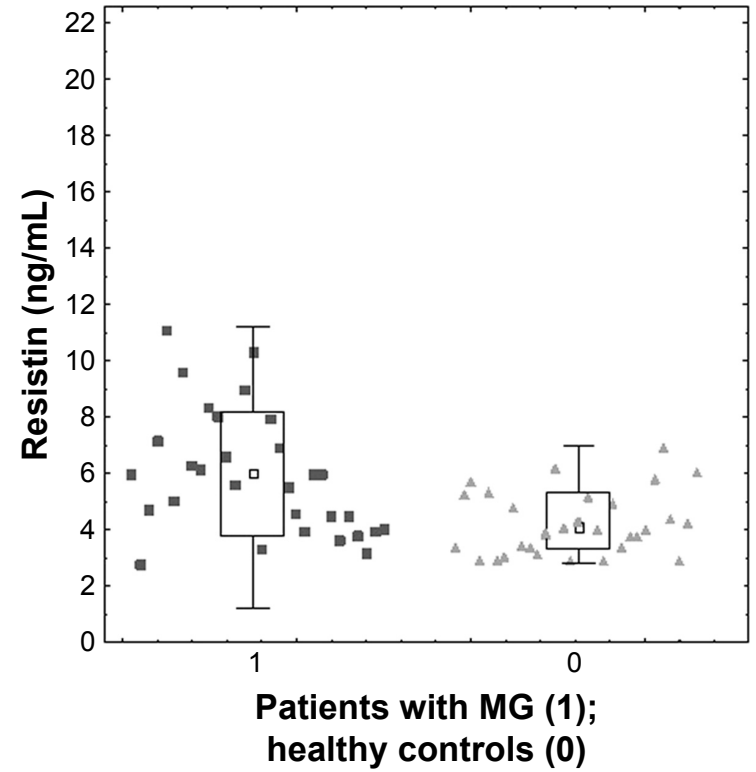

口Median $\square 25 \%-75 \%$ I Min-Max

Figure I Serum levels of adiponectin and resistin in different groups - patients with MG vs the healthy control group. Statistical comparisons were made using the MannWhitney test; $p<0.05$.

Abbreviation: MG, myasthenia gravis. 


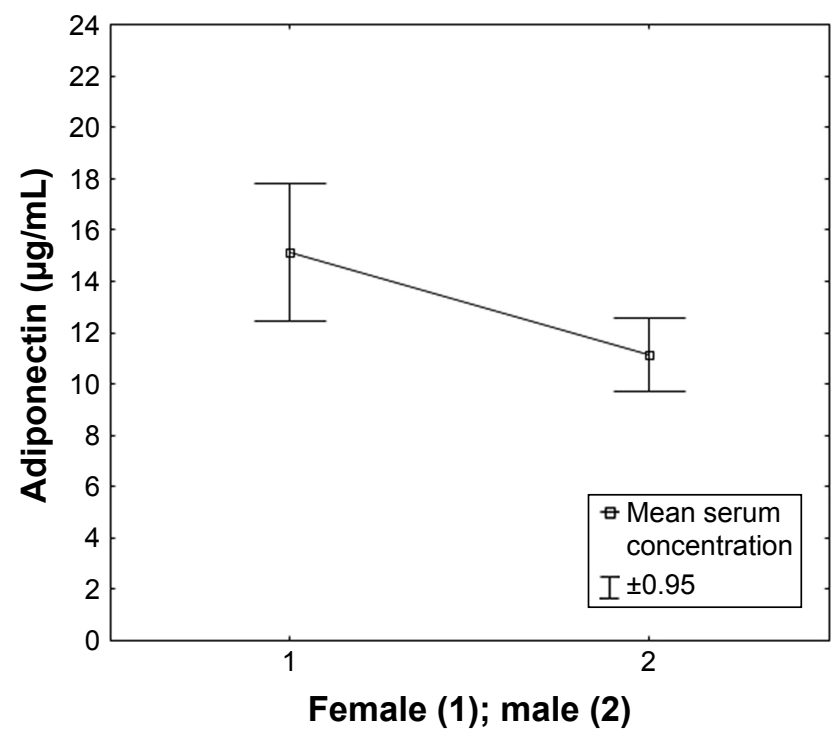

Figure 2 Serum concentration of adiponectin in patients with MG classified based on gender.

Abbreviation: MG, myasthenia gravis.

and the time after thymectomy $(p<0.001 ; r=0.6936)$. No statistically significant correlations were found for omentin concentrations in relation to the selected clinical factors. Table 7 shows the correlations obtained in the study.

Considering the selected clinical factors, a post hoc analysis was performed and significant changes were observed in the concentration of leptin. It was found that serum leptin concentration was statistically significantly higher in patients with a disease duration of over 10 years $(p<0.001)$. Similar results were obtained in patients treated with

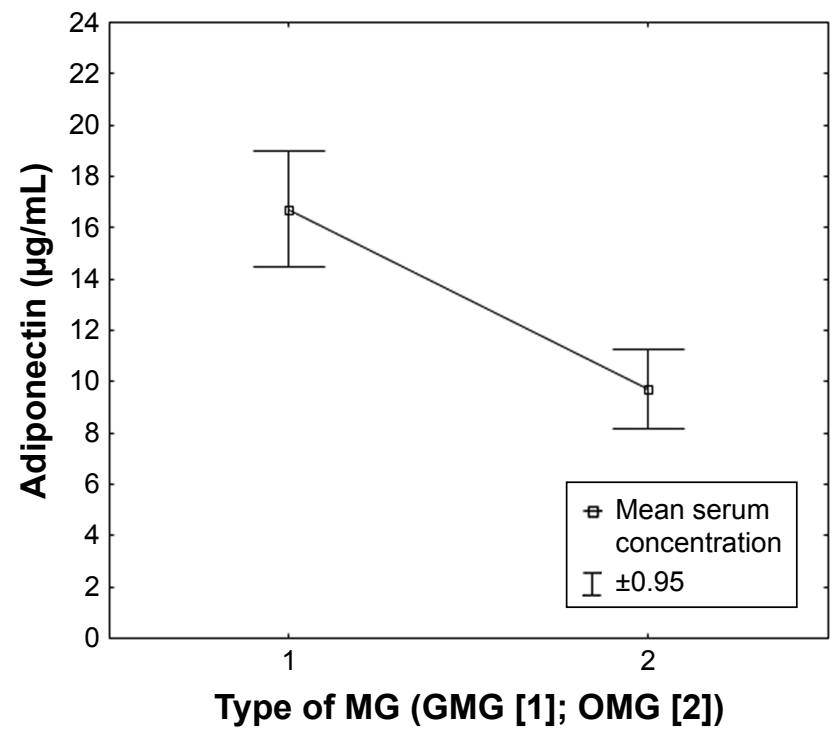

Figure 3 Serum concentration of adiponectin in patients classified based on the type of MG.

Abbreviations: GMG, general MG; OMG, ocular MG; MG, myasthenia gravis.

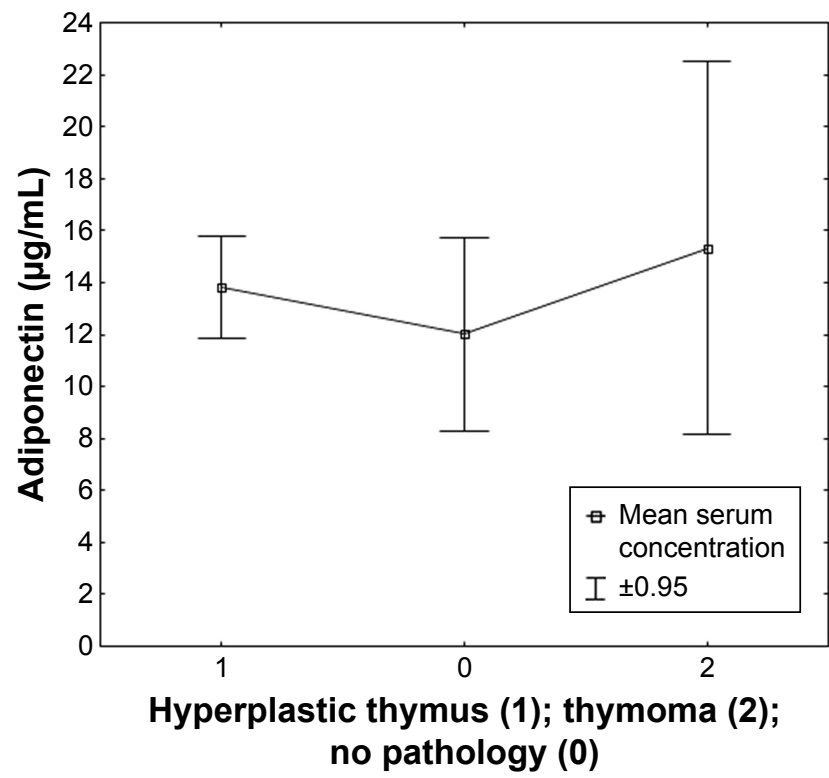

Figure 4 Serum concentration of adiponectin in patients classified based on the presence or absence of thymus pathology.

acetylcholinesterase inhibitors for a duration of over 10 years $(p<0.001)$. It was observed that leptin concentration was statistically significantly higher in patients with diagnosed mediastinal pathology in the form of hyperplastic thymus than in patients without confirmed pathology $(p<0.001)$. The results of the analysis are shown in Figures 7-9.

No relationship was observed between the elevated levels of the analyzed adipokines and the use of azathioprine. The concentrations of selected adipokines did not differ significantly between patients treated with acetylcholinesterase

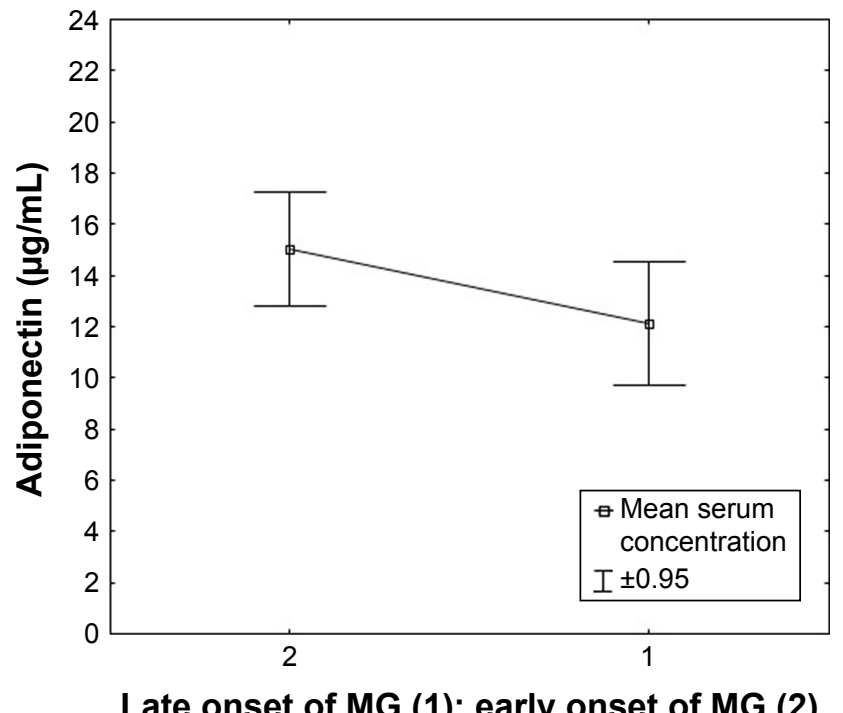

Figure $\mathbf{5}$ Serum concentration of adiponectin in patients classified based on the age at onset of MG.

Abbreviation: MG, myasthenia gravis. 


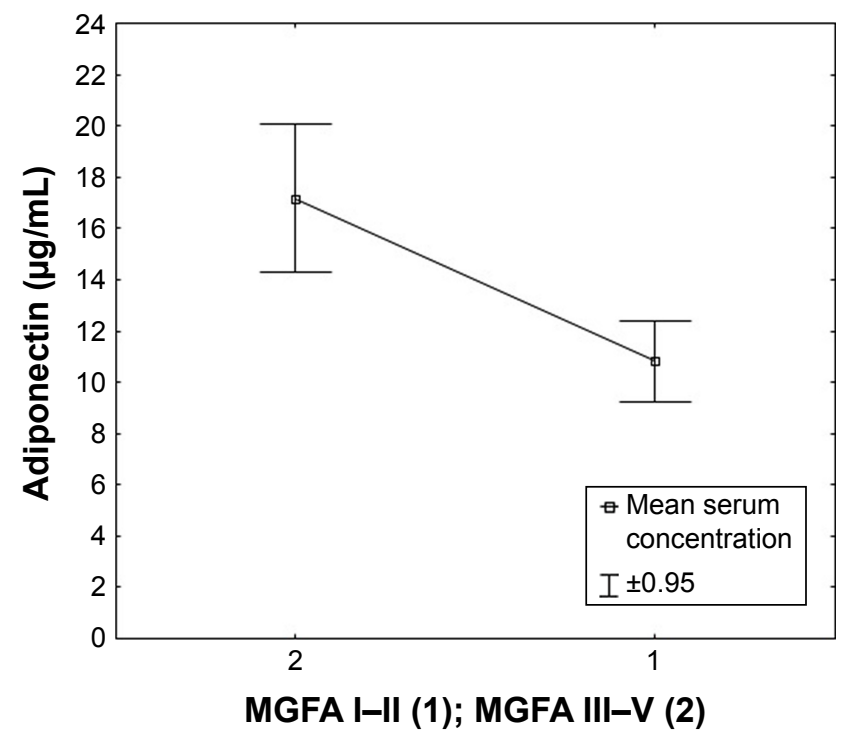

Figure 6 Serum concentration of adiponectin in patients classified based on the severity of MG.

Abbreviations: MGFA, the Myasthenia Gravis Foundation of America; MG, myasthenia gravis.

inhibitors and those treated with corticosteroids $(p=0.724)$. Similar results were obtained by analyzing patients treated with small doses of corticosteroids. The doses did not have a statistically significant effect on changes in the concentrations of the analyzed adipokines $(p=0.229)$.

\section{Discussion}

Currently, little is known about the effect of adipokines on the course of MG. Only a few exploratory studies have investigated this issue. In their study, Zhang et al showed that serum resistin levels were elevated in patients with GMG symptoms and thymoma, which correlated with the severity of the disease. Resistin was selected by those authors as a potential inflammatory marker in the course of MG. ${ }^{13}$

Two subsequent studies were related to patients undergoing glucocorticoid therapy in the course of MG. In the first study, Braz et al noted lower resistin levels and higher leptin concentrations in patients with MG treated with high doses of steroids as compared to the control group. No differences were observed in the plasma levels of adiponectin. Similar

Table 6 Post hoc analysis of changes in adiponectin concentration levels in the blood serum of patients with MG in relation to the duration of treatment with acetylcholinesterase inhibitors

\begin{tabular}{lll}
\hline $\begin{array}{l}\text { Group (according } \\
\text { to the MGFA) }\end{array}$ & MGFA I & MGFA IIa \\
\hline MGFA IIlb & $p=0.003$ & $p=0.009$ \\
\hline
\end{tabular}

Abbreviations: MG, myasthenia gravis; MGFA, the Myasthenia Gravis Foundation of America. results were obtained by Braz et al in another study, after an additional assessment of the adverse effects of long-term steroid therapy on the development of osteoporosis. ${ }^{31}$

The potential contribution of selected adipokines has been the subject of observation and research with respect to many diseases, particularly those with autoimmune etiology. This also applies to other neurological disorders, such as multiple sclerosis (MS) or vascular diseases of the central nervous system where significantly more reports are available. A study by Natarajan et al showed that in patients with different subtypes of MS and clinically isolated syndrome, adipsin had a predictive potential as a neurodegeneration biomarker. The obtained data correlated with the disease activity, the Expanded Disability Status Scale, and the volume of changes in magnetic resonance imaging. ${ }^{32}$ The role of adipokines in the regulation of immune responses in MS was also found in other studies. However, very little is known about their effect on MS.

The role of adipokines is more complicated in patients with ischemic stroke. Gorgui et al in their meta-analysis indicated that the relationship between adiponectin and the presence of atherosclerotic plaques in the carotid arteries depended on the duration, severity, and nature of the underlying disease. Increased levels of adiponectin are likely to be associated with an increased risk of ischemic stroke. The results of the meta-analysis of mortality remain ambiguous. ${ }^{33}$

In our study, we observed that adiponectin levels in the serum of patients with MG were directly proportional to the occurrence of the generalized form of the disease and the severity of generalized symptoms of muscular fatigue (MGFA class IIIb). Significantly higher serum adiponectin levels were found in patients receiving higher doses of acetylcholinesterase inhibitors. A relationship between higher adiponectin levels and IL-33 level in the serum of patients with $\mathrm{MG}$ was also observed. Among patients with longer disease duration and longer time of treatment with acetylcholinesterase inhibitors, significantly higher concentrations of both leptin and resistin were observed in those presenting with neurological symptoms. The concentration of resistin was directly proportional to the dose of drugs used and the level of inflammatory markers. Visfatin concentrations in the blood serum of patients with MG showed a correlation with the concentration of inflammatory markers, the severity of clinical symptoms of the generalized form of the disease, and a higher level of AChR antibodies. All these parameters were augmented in patients with MG and the increase was statistically significant in most groups of patients. 
Table 7 Selected correlations between adiponectin, leptin, visfatin, and resistin concentrations in the serum of patients with MG depending on clinical factors characterizing the course of the disease

\begin{tabular}{|c|c|c|c|c|c|c|c|c|}
\hline \multirow[t]{2}{*}{ Clinical factor } & \multicolumn{2}{|c|}{$\begin{array}{l}\text { Adiponectin } \\
(\mu \mathrm{g} / \mathrm{mL})\end{array}$} & \multicolumn{2}{|c|}{$\begin{array}{l}\text { Leptin } \\
(\mathrm{ng} / \mathrm{mL})\end{array}$} & \multicolumn{2}{|c|}{$\begin{array}{l}\text { Visfatin } \\
(\mathrm{ng} / \mathrm{mL})\end{array}$} & \multicolumn{2}{|c|}{$\begin{array}{l}\text { Resistin } \\
(\mathrm{ng} / \mathrm{mL})\end{array}$} \\
\hline & $\boldsymbol{r}$ & $p$-value & $\boldsymbol{r}$ & $p$-value & $\boldsymbol{r}$ & $p$-value & $\boldsymbol{r}$ & $p$-value \\
\hline Disease duration & ns & ns & 0.6313 & $p<0.001$ & ns & ns & ns & ns \\
\hline AChR antibody level & ns & ns & ns & ns & 0.3429 & $p=0.015$ & ns & ns \\
\hline ACh-esterase inhibitor dose & 0.3438 & $p=0.014$ & 0.3272 & $p=0.02$ & ns & ns & 0.4102 & $p=0.003$ \\
\hline Form of MG & 0.5983 & $p<0.001$ & 0.299 & $p=0.035$ & 0.3125 & $p=0.027$ & ns & ns \\
\hline Gender & 0.3383 & $p=0.016$ & ns & ns & ns & ns & ns & ns \\
\hline Treatment duration & ns & ns & 0.8062 & $p<0.001$ & ns & ns & 0.3147 & $p=0.026$ \\
\hline MG-QOL-I5 score & 0.3884 & $p=0.005$ & ns & ns & ns & ns & ns & ns \\
\hline MG Composite score & 0.3117 & $p=0.028$ & ns & ns & ns & ns & ns & ns \\
\hline BMI & -0.3967 & $p=0.004$ & ns & ns & ns & ns & ns & ns \\
\hline Time duration after thymectomy & ns & ns & 0.6936 & $p<0.001$ & ns & ns & ns & ns \\
\hline Apokamnosis & 0.5329 & $p<0.001$ & 0.3495 & $p=0.013$ & 0.3417 & $p=0.015$ & ns & ns \\
\hline MGFA IIIb & 0.4514 & $p=0.001$ & ns & ns & ns & ns & ns & ns \\
\hline IL-33 & 0.6591 & $p<0.001$ & ns & ns & ns & ns & ns & ns \\
\hline CRP & ns & ns & ns & ns & 0.4966 & $p<0.001$ & 0.3125 & $p=0.006$ \\
\hline
\end{tabular}

Abbreviations: MG, myasthenia gravis; AChR, acetylcholine receptor; ACh, acetylcholine; MG-QOL-I5, Myasthenia Gravis Quality of Life I5; BMI, body mass index; MGFA, the Myasthenia Gravis Foundation of America; CRP, C-reactive protein; ns, nonsignificant.

Changes in the concentrations of adipokines in the blood serum of patients with MG suggest their possible contribution to the pathomechanism of the disease. Adiponectin has mainly an anti-inflammatory effect; hence, according to our observations, high level of adiponectin in patients with severe symptoms of the disease may be indicative of the body's response to exacerbation of the disease. The obtained results allow us to present some hypotheses. The problem seems to be bidirectional and poses the following question - can we postulate the role of adiponectin as a factor involved in the pathogenesis of the disease or rather treat it as a biomarker? Both solutions seem to be probable; however, our observations at this stage of the study rather support the role of adiponectin as a biomarker. It should be emphasized that these are only preliminary assumptions and further studies are needed in this respect.

Resistin, with its mainly proinflammatory effect, also showed a positive correlation between clinical symptoms of $\mathrm{MG}$ and an increase in its concentration in relation to healthy persons. Our results confirm a significant relationship between a higher concentration of leptin in patients with a disease duration and the length of treatment with acetylcholinesterase inhibitors of over 10 years. Therefore, it should be verified whether the elevated leptin level is the result of the disease itself, its natural course, or the long-term impact of the drug use. The proinflammatory nature of leptin may suggest that the duration of the disease could negatively affect disease control in selected patients. Further observation is also required with respect to the assessment of adipokine concentration that depends on the presence of hyperplastic thymus or thymoma in the mediastinum. The obtained data indicate the existence of a relationship between the level of leptin in the blood serum of patients and the presence of hyperplastic thymus. Further studies are warranted to assess changes in adipokine concentration levels in the case of surgical treatment of patients. Only in the case of visfatin, the relationship between visfatin and the level of AChR antibodies was observed. It may indicate the lack of a direct

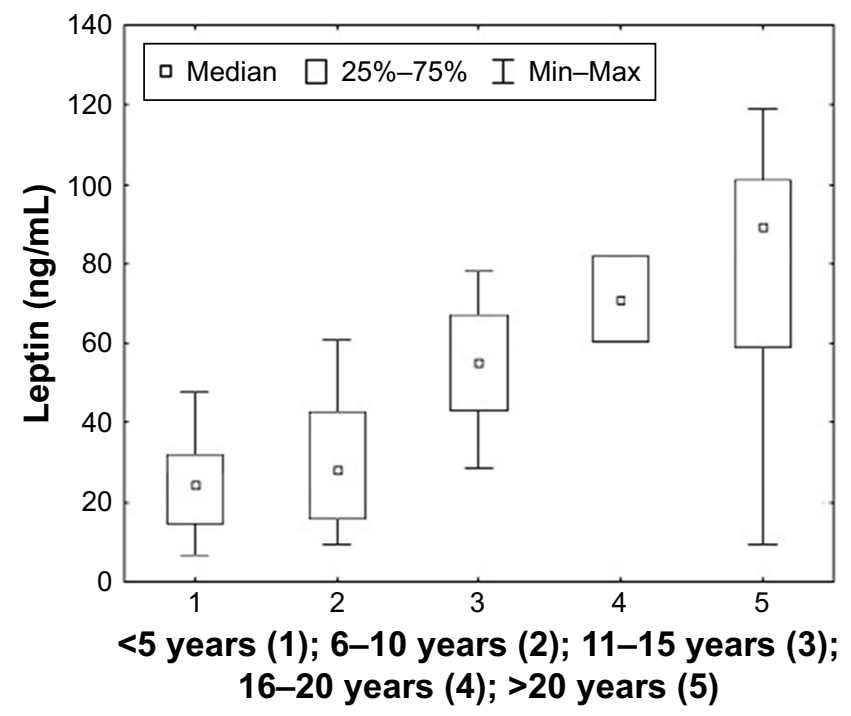

Figure 7 The concentration of leptin in relation to the duration of the disease in patients with MG. Statistical analysis was performed using the Kruskal-Wallis test. Abbreviation: MG, myasthenia gravis. 


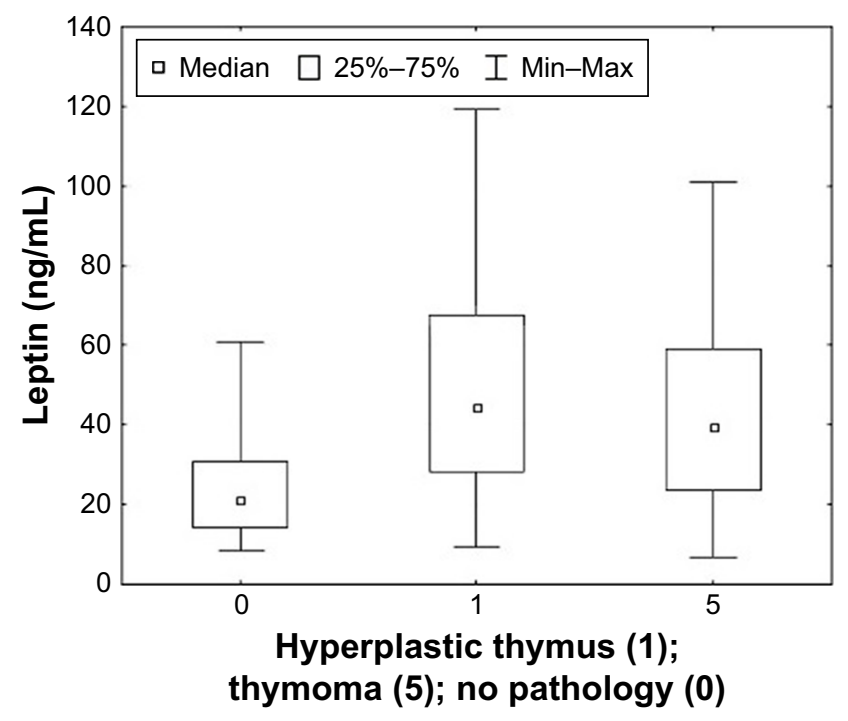

Figure 8 Leptin concentration in relation to the diagnosed presence or absence of pathology in the mediastinum in patients with MG. Statistical analysis was performed using the Kruskal-Wallis test.

Abbreviation: MG, myasthenia gravis.

effect of changes in the concentration of adipokines on the function of the neuromuscular junction.

A parallel study on the role of IL-33 in the etiopathogenesis of MG conducted in our center revealed the possibility of a correlation between the concentration of this IL and the severity of clinical symptoms of MG. In the present study, we performed an additional analysis, based on which we observed that IL-33 levels showed a strong relationship with adiponectin levels in the serum of patients with $\mathrm{MG} .^{34}$

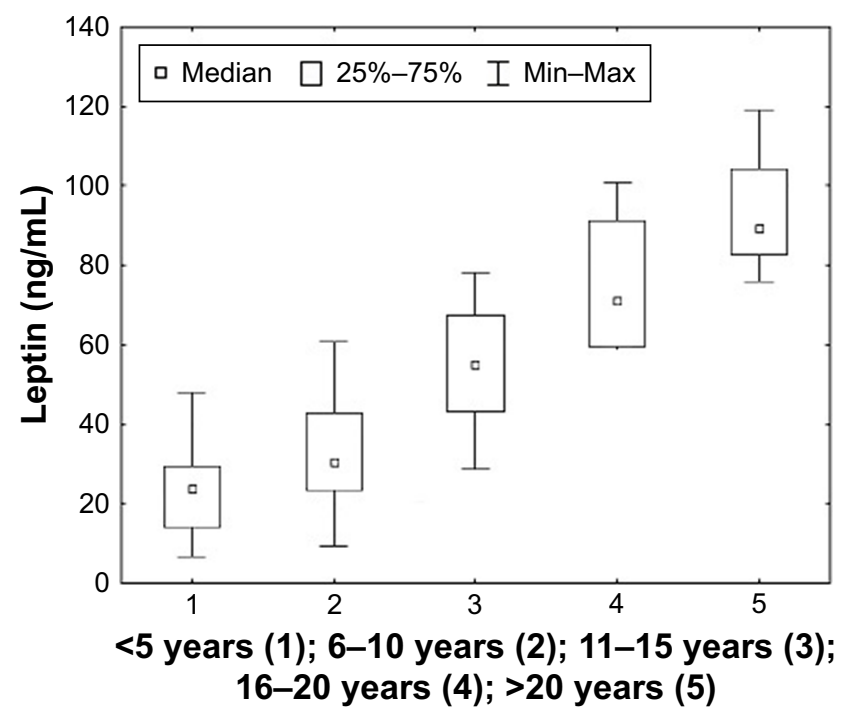

Figure 9 Leptin concentration in relation to the duration of time after thymectomy in patients with MG. Statistical analysis was performed using the Kruskal-Wallis test. Abbreviation: MG, myasthenia gravis.

\section{Conclusion}

We are aware of the limitations of our study primarily related to a small group of patients. In addition, performing only single tests cannot reflect the mechanisms occurring in the course of the disease. The current study is a pilot trial, and our observations should be continued and further investigated.

\section{Acknowledgment}

This study was supported by the Medical University of Silesia.

\section{Disclosure}

The authors report no conflicts of interest in this work.

\section{References}

1. Meriggioli M, Sanders D. Autoimmune myasthenia gravis: emerging clinical and biological heterogeneity. Lancet Neurol. 2009;8(5): 475-490.

2. Wang L, Zhang Y, He M. Clinical characteristics of patients with myasthenia gravis associated with other autoimmune diseases. Chin J Contemp Neurol Neurosurg. 2014;14(10):873-877.

3. Vincent A, Palace J, Hilton-Jones D. Myasthenia gravis. Lancet. 2001;357:2122-2128.

4. Rodriguez Cruz PM, Al-Hajjar M, Huda S, et al. Clinical features and diagnostic usefulness of antibodies to clustered acetylcholine receptors in the diagnosis of seronegative myasthenia gravis. JAMA Neurol. 2015;72:642-649.

5. Zhang B, Tzartos J, Belimezi M, et al. Autoantibodies to lipoproteinrelated protein 4 in patients with double-seronegative myasthenia gravis. Arch Neurol. 2012;69(4):445-451.

6. Higuchi O, Hamuro J, Motomura M, Yamanashi Y. Autoantibodies to low-density lipoprotein receptor-related protein 4 in myasthenia gravis. Ann Neurol. 2011;69(2):418-422.

7. Pevzner A, Schoser B, Peters K, et al. Anti-LRP4 autoantibodies in AChR- and MuSK-antibody-negative myasthenia gravis. J Neurol. 2012;259(3):427-435.

8. Andersen JB, Owe JF, Engeland A, Gilhus NE. Total drug treatment and comorbidity in myasthenia gravis: a population-based cohort study. Eur J Neurol. 2014;21(7):948-955.

9. Balandina A, Lecart S, Dartevelle P, Saoudi A, Berrih-Aknin S. Functional defect of regulatory $\mathrm{CD} 4(+) \mathrm{CD} 25+\mathrm{T}$ cells in the thymus of patients with autoimmune myasthenia gravis. Blood. 2005;105: 735-741.

10. Thiruppathi M, Rowin J, Ganesh B, Sheng J, Prabhakar B, Meriggioli MN. Impaired regulatory function in circulating CD4(+)CD25(high) CD127(low/-) T cells in patients with myasthenia gravis. Clin Immunol. 2012;145:209-223.

11. Gradolatto A, Nazzal D, Truffault F, et al. Both Treg cells and Tconv cells are defective in the myasthenia gravis thymus: roles of IL-17 and TNF- $\alpha . J$ Autoimmun. 2014;52:53-63.

12. Diez J, Iglesias P. The role of the novel adipocyte-derived hormone adiponectin in human disease. Eur J Endocrinol. 2003;148:293-300.

13. Zhang DQ, Wang R, Li T, et al. Remarkably increased resistin levels in anti-AChR antibody-positive myasthenia gravis. J Neuroimmunol. 2015;283:7-10.

14. Ashima R, Qi Y, Singhal N. Brain adipokine action and metabolic regulation. Diabetes. 2006;55:145-154.

15. Ouchi N, Parker J, Lugus J, Walsh K. Adipokines in inflammation and metabolic disease. Nat Rev Immunol. 2011;11:85-97. 
16. De Sanctis J, Zabaleta M, Bianco N, Garmendia J, Rivas L. Serum adipokine levels in patients with systemic lupus erythematosus. Autoimmunity. 2009;42(4):272-274.

17. Zhang M, Dini A, Yang X, et al. Association between serum/plasma adiponectin levels and immune-mediated diseases: a meta-analysis. Arch Dermatol Res. 2017;309(8):625-635.

18. Li H, Zhang T, Leng R, Li X, Li X, Pan H. Plasma/serum leptin levels in patients with systemic lupus erythematosus: a meta-analysis. Arch Med Res. 2015;46(7):551-556.

19. Wislowska M, Rok M, Stepien K, Kuklo-Kowalska A. Serum leptin in systemic lupus erythematosus. Rheumatol Int. 2008;28(5):467-473.

20. Almehed K, d'Elia H, Bokarewa M, Carlsten H. Role of resistin as a marker of inflammation in systemic lupus erythematosus. Arthritis Res Ther. 2008;10(1):15.

21. Huang Q, Tao S, Zhang Y, et al. Serum resistin levels in patients with rheumatoid arthritis and systemic lupus erythematosus: a meta-analysis. Clin Rheumatol. 2015;34(10):1713-1720.

22. Shehzad A, Iqbal W, Shehzad O, Lee YS. Adiponectin: regulation of its production and its role in human diseases. Hormones. 2012;11:8-20.

23. La Cava A, Matarese G. The weight of leptin in immunity. Nat Rev Immunol. 2004;4:371-379.

24. Filkova M, Haluzik M, Gay S, Senolt L. The role of resistin as a regulator of inflammation: implications for various human pathologies. Clin Immunol. 2009;133(2):157-170.

25. Moschen AR, Kaser A, Enrich B, et al. Visfatin, an adipokine with proinflammatory and immunomodulating properties. J Immunol. 2007; 178(3):1748-1758

26. Hernández-Díaz A, Arana-Martínez JC, Carbó R, Espinosa-Cervantes R, Sánchez-Muñoz F. Omentina: papel en la resistencia a la insulina, inflamación y protección cardiovascular [Omentin: role in insulin resistance, inflammation and cardiovascular protection]. Arch Cardiol Mex. 2016;86:233-243. Spanish [with English abstract].
27. Burn T, Conaway M, Cutter G, Sanders D. Construction of an efficient evaluative instrument of myasthenia gravis: the MG composite. Muscle Nerve. 2008;38:1553-1562.

28. Wolfe G, Herbelin L, Nations S, Foster B, Bryan W, Barohn R. Myasthenia gravis activities of daily living profile. Neurology. 1999;52(7): 1487-1489.

29. Burns T, Conaway M, Cutter G, Sanders D; Muscle Study Group. Less is more, or almost as much: a 15-item quality-of-life instrument for myasthenia gravis. Muscle Nerve. 2008;38(2):957-963.

30. Jaretzki A, Barohn R, Ernstoff R, et al. Myasthenia gravis: recommendations for clinical research standards. Task Force of the Medical Scientific Advisory Board of the Myasthenia Gravis Foundation of America. Ann Thorac Surg. 2000;70:327-334.

31. Braz N, Rocha N, Vieira É, et al. Negative impact of high cumulative glucocorticoid dose on bone metabolism of patients with myasthenia gravis. Neurol Sci. 2017;38(8):1405-1413.

32. Natarajan R, Hagman S, Hämälainen M, et al. Adipsin is associated with multiple sclerosis: a follow-up study of adipokines. Mult Scler Int. 2015;2015:371734.

33. Gorgui J, Gasbarrino K, Georgakis M, et al. Circulating adiponectin levels in relation to carotid atherosclerotic plaque presence, ischemic stroke risk, and mortality: a systematic review and meta-analyses. Metabolism. 2017;69:51-66.

34. Rozmilowska I, Adamczyk-Sowa M. What is the role of interleukin 33 and ST2 receptor in myasthenia gravis? J Neuroimmunol. 2018;315:50-57.
Therapeutics and Clinical Risk Management

\section{Publish your work in this journal}

Therapeutics and Clinical Risk Management is an international, peerreviewed journal of clinical therapeutics and risk management, focusing on concise rapid reporting of clinical studies in all therapeutic areas, outcomes, safety, and programs for the effective, safe, and sustained use of medicines. This journal is indexed on PubMed Central, CAS,

\section{Dovepress}

EMBase, Scopus and the Elsevier Bibliographic databases. The manuscript management system is completely online and includes a very quick and fair peer-review system, which is all easy to use. Visit http://www.dovepress.com/testimonials.php to read real quotes from published authors.

Submit your manuscript here: http://www.dovepress.com/therapeutics-and-clinical-risk-management-journal 